\title{
Can origin of the 2400-year cycle of solar activity be caused by solar inertial motion?
}

\author{
I. Charvátová \\ Geophysical Institute AS CR, Boční II, 14131 Praha 4, Czech Republic \\ Received: 30 September 1999 / Revised: 14 January 2000 / Accepted: 17 January 2000
}

\begin{abstract}
A solar activity cycle of about 2400 years has until now been of uncertain origin. Recent results indicate it is caused by solar inertial motion. First we describe the 178.7-year basic cycle of solar motion. The longer cycle, over an 8000 year interval, is found to average 2402.2 years. This corresponds to the Jupiter/ Heliocentre/Barycentre alignments $(9.8855 \times 243)$. Within each cycle an exceptional segment of 370 years has been found characterized by a looping pattern by a trefoil or quasitrefoil geometry. Solar activity, evidenced by ${ }^{14} \mathrm{C}$ tree-ring proxies, shows the same pattern. Solar motion is computable in advance, so this provides a basis for future predictive assessments. The next 370year segment will occur between AD 2240 and 2610.
\end{abstract}

Key words: Solar physics (celestial mechanics)

\section{Introduction}

To find a cause of solar variability is a key task for solar physics. It is an important subject also for geophysics due to the influence of this variability on the Earth's climate. Over recent centuries, enormous efforts have been given to this problem. In recent decades satellites have provided much data and in particular the observations of the satellite $\mathrm{SOHO}$ provides a more reliable view of the solar interior.

It is now generally accepted that the two most prominent long-term cycles are permanently present in solar activity: a cycle of about $200(160-210)$ years and a cycle of about $2400(2200-2600)$ years, see e.g. Suess (1980), Sonett and Finney (1991), Damon and Linick (1986), Damon et al. (1989), Damon and Sonett (1990), Hood and Jirikowic (1991), etc. These studies have been made on the basis of indirect, proxy ${ }^{14} \mathrm{C}$ record in tree rings. A scatter of the cycle lengths observed is probably due to the mixed quality of the records, the different parts of the record being processed, and the various methods of spectral analysis used, etc. It is difficult to observe this periodicity of 200 years because the record of sunspot numbers covers only 300 years. It is easier to observe a periodicity of about 100 years (Gleissberg cycle) connected with the amplitude modulation of the sunspot number series. An origin of the prominent, and permanently present, period of about 2400 years in solar activity has still not been settled (i.e., is it solar, geomagnetic, or extraheliospheric) or even enigmatic; the underlying 'forcing' being so far unknown. "The 2400-year period appears to be stationary" according to Damon and Linick (1986).

The solar inertial motion (i.e., the motion of the Sun around the centre of mass of the solar system) is the central phenomenon of the solar system, caused by varying positions, predominantly, of the giant planets (Fairbridge and Sanders, 1987). The contribution by the inner planets is minute. The varying positions of the giant planets [Jupiter (J), Saturn (S), Uranus (U), Neptune (N)] force the Sun to move inside a circular area which has a diameter of $0.02 \mathrm{AU}$ (astronomical unit) or $3 \cdot 10^{6} \mathrm{~km}$, see Fig. 1. This is negligible in comparison with the size of the solar system, but it is very significant with respect to the size of the Sun. The diameter of the area in which the Sun moves represents 4.4 solar radii. The Sun moves with a velocity between 9 and $16 \mathrm{~m} \cdot \mathrm{s}^{-1}$, i.e. 30 and $60 \mathrm{~km} \cdot \mathrm{h}^{-1}$. This solar motion is computable in advance, a great advantage that opens up the possibility of establishing predictive assessments of solar activity.

To understand the 2402-year cycle, it is necessary to describe first and explain the basic cycle of solar motion, the cycle of 178.7 years and its relations to solar activity behaviour over recent centuries. Both cycle values have been found to correspond to the intervals between the exceptional patterns in solar motion. The values represent, in both cases, the mean of the dispersion intervals of the prominent cycles found in solar activity mentioned. 


\section{SO (1600-2060)}

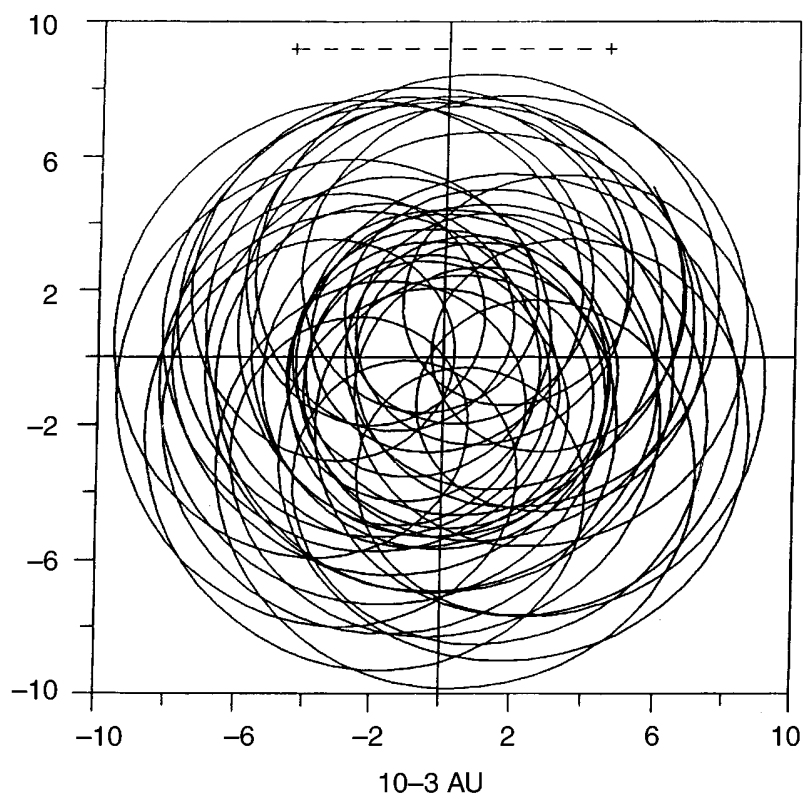

Fig. 1. The orbit of the centre of the Sun around the centre of mass of the solar system (in units of $10^{-3} \mathrm{AU}$, astronomical unit $=149 \cdot 10^{6} \mathrm{~km}$ ), for further details see Fig. 2. The dashed horizontal abscissa in the upper part of the figure represents the diameter of the Sun

\section{The first basic cycle of 178.7 years in solar motion and its response in terms of solar activity}

The first basic cycle of solar inertial motion, the cycle of 178.7 years, was found by Jose (1965) in a repetition of solar motion characteristics computed between 1653 and 2060, and most important the time derivative of the Sun's angular momentum was found. The cycle was confirmed by Fairbridge and Shirley (1987) since 760 AD, and by Fairbridge and Sanders (1987) since 777 AD. Jakubcová and Pick (1987) verified the 178.7 year cycle as the basic period of solar motion periodicities (see their Fig. 1). These periods have been found as its higher harmonics [also Fairbridge, 1997, e.g. $28 \times 178.7=5004.5(252 \times \mathrm{JS} ; 24 \times 208.5$ year VJU $)]$ and mostly correspond to the orbital periods of the giant planets [i.e. the periods of $80-90(\mathrm{U}), 60,45(\mathrm{SN}), 35$ (SU), 30 (S), 13.8 (JU), 12.8 (JN), 11.9 (J), 10 (JS/2)].

The Sun moves in the plane of the solar system, so this action is essentially planar ( $Z$-coordinates have been neglected for initial studies). Therefore, besides the motion characteristics, the geometry of the solar orbit should be most carefully considered. Eventually, this geometrical approach did provide a key for the solution of the solar motion - solar activity relationship.

A key to solution consists in the separation of solar motion into two basic orbital types (Charvátová, 1988, 1990a, b), see Fig. 2: the ordered (according to JS motion order, $117.3^{\circ}, 19.86$ years in a trefoil) and the disordered. The Sun enters into the orbital trefoils with a spacing, on average, of 178.7 years and moves along a trefoil (in one loop) in about 50 years (10 years), respectively. While the trefoil orbits are, after a rotation, nearly the same, the disordered orbits differ from one to another. After separation, the solar motion itself was discernible and fixed in time as a precise and homogeneous basis suitable for solar-terrestrial studies. Then, it has been possible to observe separated solar motion from a particular view point. The trefoil intervals which recur in regular steps of 178.7 years can be taken and employed as the exceptional and stable pattern of solar motion.

A response to solar motion has been observed in solar activity. In fact, it was noticed that the intervals of disordered solar motion coincide with particular prolonged minima in solar activity, such as the Wolf, Spörer, Maunder and Dalton minima of this millennium.

The results obtained for mutual relationships between solar motion and solar activity during the latest, basic 178.7 year cycle are summarized in Fig. 3a-d. If solar motion is really a cause of solar variability, then the motion along the same (trefoil) orbit should create the same series of sunspot cycles. In fact, the Sun moving along the same orbits during the trefoil intervals of the eighteenth century (1734-1785) and the twentieth century (1913-1964) (taken from minimum to minimum of the sunspot cycles), created nearly the same sets of five sunspot cycles (Charvátová, 1990b, 1995a, 1997a, b): cycles -1 to 3 and cycles 15 to 19 . Small deviations can be mainly ascribed to lower quality of sunspot numbers in the eighteenth century. Unfortunately, the Wolf number series is not homogeneous. It can be taken as reliable only since about 1850 . Earlier, it is of lower quality and before 1749 not even the monthly data are available. This is not sufficient to confirm an exact response of the solar trefoil motion in the Wolf series. The highest and the only significant coefficient of correlation between the successive groups of five sunspot cycles since 1700 and the series from the trefoil intervals is that $(r=0.81)$ between the two series of five cycles from both trefoil intervals. The basic cycle of 179 years in those two phenomena, the mutual relation between solar motion and solar activity thus became evident (Fig. 3d).

It was shown (Charvátová, 1995b, c, 1997a) that the lengths of five sunspot cycles (15-19) created by the Sun moving along the trefoil orbit where motion along one motion loop lasts 10 years (see Fig. 2, top), is stable and equal to $10.1(\sim \mathrm{JS} / 2)$ years. The more precise length of the cycles -1 to 3 cannot be calculated, mainly due to the lack of monthly data before 1749 . However, the mean length of the respective five cycles is also about 10 years. In the surrounding and intermediate intervals, the lengths of sunspot cycles are variable and generally greater (Fig. 3a). The dominant period of 10.1 (JS/2) years was detected in sunspot numbers from the both trefoil intervals (Charvátová, 1990b, Fig. 4), in contrast to the longer dominant periods found for the other intervals, up to $11.9(\mathrm{~J})$ years detected for the Dalton minimum in solar activity (1790-1843) (Charvátová and Střeštík, 1994). Rabin et al. (1986) and Wilson (1988) found that solar behaviour is bimodal, with modes being 10.1 and 11.9 years. 

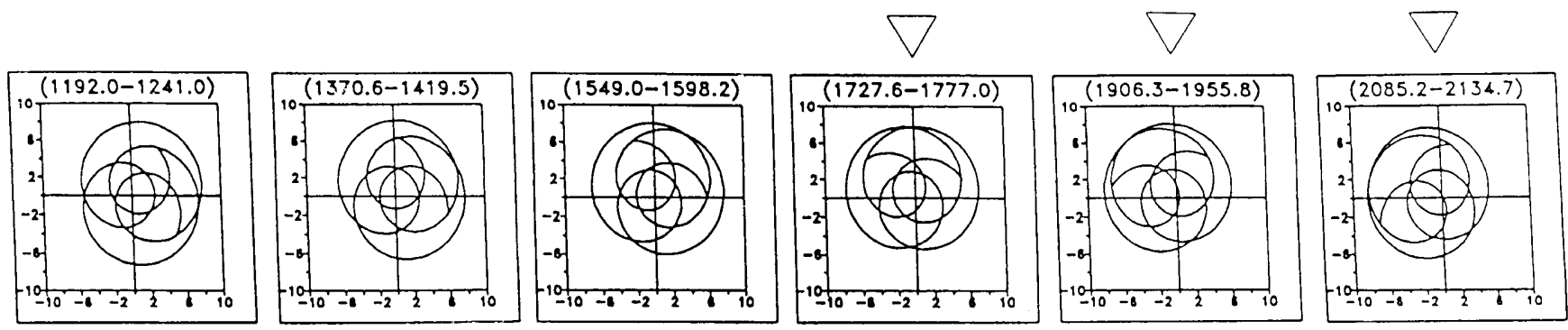

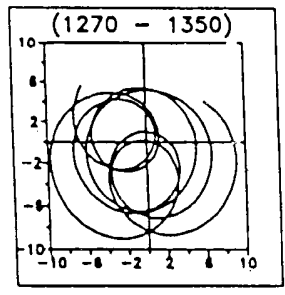

WOLF

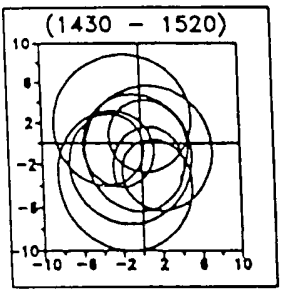

SPÖRER

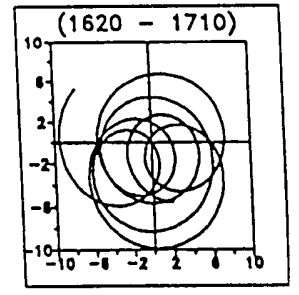

MAUNDER

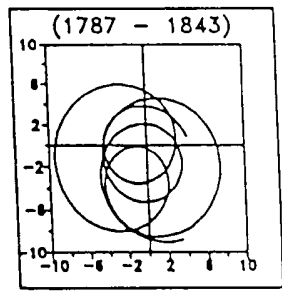

DALTON

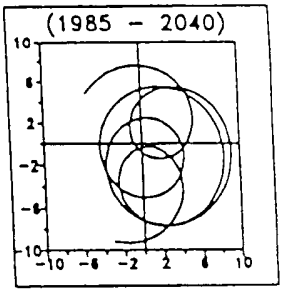

NEXT?
Fig. 2. The orbit of the centre of the Sun around the centre of mass of the solar system (in units of $10^{-3} \mathrm{AU}$ ) separated into two basic types, the ordered (in a JS-trefoil) (top) and the disordered (bottom). The area in which the Sun moves has a diameter of $0.02 \mathrm{AU}$ or $4.4 r_{s}$, this being the solar radius, or $3 \cdot 10^{6} \mathrm{~km}$. The most disordered sections of the intervals lying between the trefoils are plotted. The Sun enters into the trefoils with a periodicity of 178.7 years, on the average (see the times, years at the top of the respective figures). The value represents

\section{The second basic cycle in solar motion: 2402 years, and its response in the proxy solar activity record $\left({ }^{14} \mathrm{C}\right.$ in tree rings)}

On the very long (millennial) time scale only indirect, proxy ${ }^{14} \mathrm{C}$ records of solar activity (radiocarbon in treerings) are available. Radiocarbon production in the atmosphere and consequently in tree-rings, is owing to a cosmic ray flux which has significantly varied, being in inverse relationship with solar activity. The radiocarbon record in tree-rings thus provides a reliable archive of past solar activity.

The regularity of the 178.7-year cycle is sometimes disturbed: e.g. between $158 \mathrm{BC}$ and $208 \mathrm{AD}$, the first 178.7-year basic cycle (the interval between the two consecutive orbital trefoils) was twice shortened to 159 years and during the intermediate intervals the Sun moved along an orbit that is not too far from a trefoil. These 370-year segments of exceptional and nearly stable motion of the Sun (along a trefoil to quasitrefoil orbit) have been found to recur in steps of 2402 years, the mean value being 2402.2 years.

Figure 4 displays the last three such segments: the first from $158 \mathrm{BC}$ to $208 \mathrm{AD}$, the second from $2561 \mathrm{BC}$ to $2193 \mathrm{BC}$ and the third from $4964 \mathrm{BC}$ to $4596 \mathrm{BC}$. The solar orbits are the same in all three cases, if we imagine them after a rotation. Further back in the past, the Sun moved along the same orbital pattern between the years 7366 BC and 6998 BC. The next such segment will occur between the years $2242 \mathrm{AD}$ and 2610 AD. the first basic cycle of solar motion. While the trefoils are nearly identical (after a rotation), the disordered orbits differ one from the other. The Wolf, Spörer, Maunder and Dalton prolonged minima of solar activity coincide with the intervals of disordered solar motion. The Sun moves along a trefoil (along one of the loops), over 50 (10) years, respectively. The two latest and the following trefoils are denoted by triangles

The strongest variations in solar activity occurred during each of the second millenia, approximately, where the longest on prolonged solar minima of Spörer (S) and Maunder (M) type occurred. The patterns of the S- and M- minima have always been observed to be different. Hood and Jirikowic (1991) note that the 2400year cycle modulates the amplitude of the cycle at about 200 (160-210) years. Charvátová $(1995 b, c)$ pointed out the coincidence of $\mathrm{S}(\mathrm{M})$ solar orbital patterns with the respective $\mathrm{S}(\mathrm{M})$ types of solar minima occurrence, (see Damon and Linick, 1986).

Note should be made here of the symmetry between the moments of the Sun's entrances into the 50-year trefoil orbits during the first millennium $(158,338,516$, $695, \ldots$, BC) and the first millennium $(159,338,516,695$, ..., AD). The centre of symmetry is the year $25 \mathrm{AD}$ (for details see Charvátová, 1995b, c). In the centre of the 2402-year cycle, smaller irregularities occur in 178.7year cycle.

The exceptional segments of 370 years that regularly recur in steps of 2402 years have served as a further tool in searching for a response to solar inertial motion in solar activity (Charvátová, 1998). The properties of the 370year segment (exceptionality and approximate stability) are found in the corresponding intervals of the proxy (radiocarbon) record and provide evidence for a solar motion response and indicate that the solar motion could be a cause of solar variability also on a millennial scale.

An analogy with a response of the 50-year trefoil orbital pattern in solar activity (high solar activity cycles with constant 10-year length) suggests that the behav- 
a)

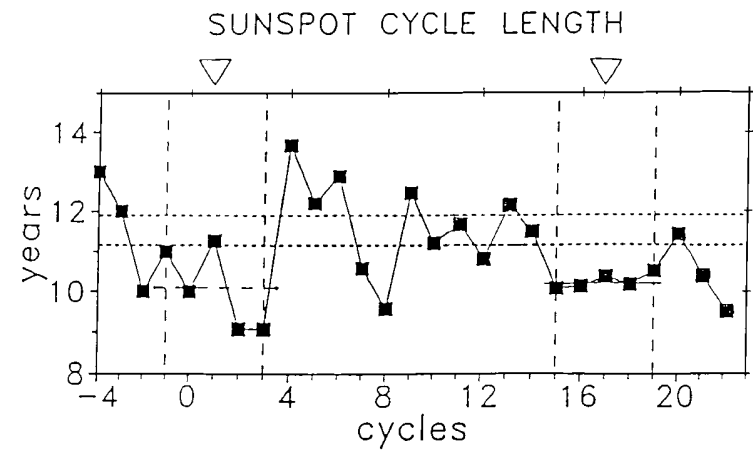

b)

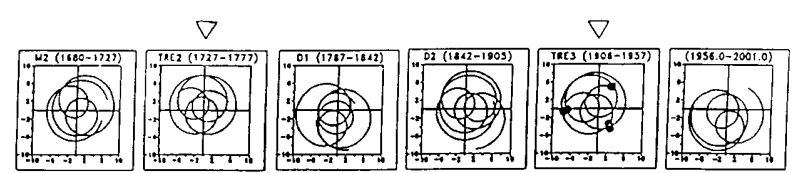

c)
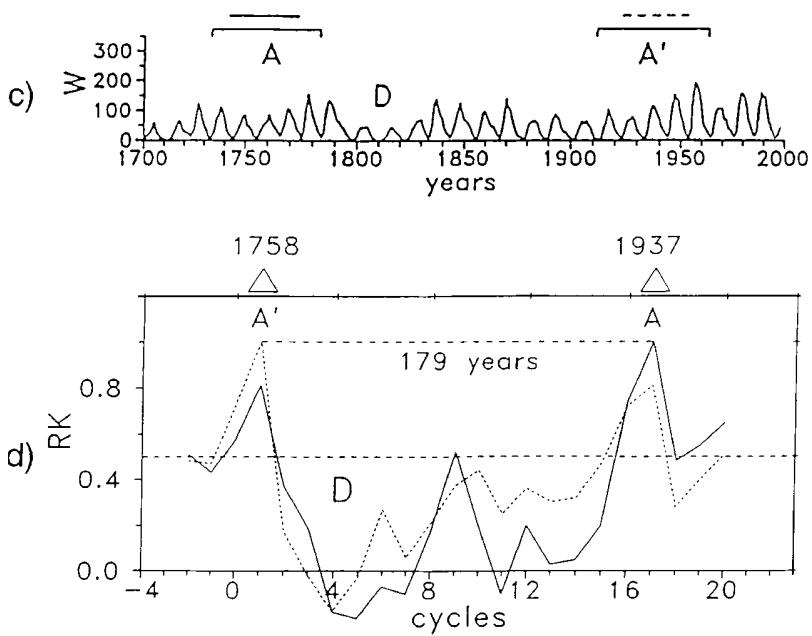

Fig. 3a-d. This figure summarizes the results obtained for solar motion - solar activity relation around the latest basic 178.7-year cycle. a The lengths of sunspot cycles since AD 1700: notice a constant cycle length equal to 10 (JS/2) years during the trefoil interval 19131964 (taken from minimum to minimum). During the previous trefoil interval (1734-1785) the cycle lengths cannot be calculated more precisely (see text). However, the mean value of cycle lengths is also 10 years there. In the surrounding and intermediate intervals, the cycle lengths are variable and mostly greater. b The solar orbit from $\mathrm{AD}$ 1680 to 2001 in sections of $50-65$ years. Two trefoil intervals are denoted by the triangles. The black circles denote the positions of the sunspot cycle maxima. c The Wolf sunspot numbers since AD 1700 . The letter $A\left(A^{\prime}\right)$ denotes the trefoil interval of the eighteenth (twentieth) century. d Coefficients of correlation between the series of five sunspot cycles that occurred during the trefoil intervals [1734 $1785(A)$ and 1913-1964 $\left(A^{\prime}\right)$ ] and the successive groups of five sunspot cycles in steps of one cycle since 1700 . The only significant coefficient $\left(r_{k}=0.81\right)$ is that between the groups of five cycles belonging to the trefoil intervals. The letter $D$ denotes the Dalton minimum of solar activity

iour of solar activity during the exceptional 370-year orbital pattern can be characterized as trefoil to quasitrefoil, or could be close to that described for a trefoil.

Figure 5 shows radiocarbon ${ }^{14} \mathrm{C}$ records for the three latest exceptional segments (the respective solar orbits are plotted in Fig. 4) together with the adjacent millennia, i.e. from $5800 \mathrm{BC}$ to $3800 \mathrm{BC}$, from $3400 \mathrm{BC}$ to 1400 $\mathrm{BC}$ and from $1000 \mathrm{BC}$ to $1000 \mathrm{AD}$. In all three cases, exceptionality and approximate stability (without prolonged minima) is seen. In this figure, local or regional data (Irish or German oaks and pine) are taken from Pearson and Quay (1993), Stuiver and Becker (1993) and Stuiver and Pearson (1993). The most stationary part of the record is seen precisely during the 370-year exceptional segments of solar motion.

Figure 6 represents the whole series of data (marine model ${ }^{14} \mathrm{C}$ ages calculated from atmospheric tree-ring data with a smoothing spline through coral data) taken from Stuiver and Braziunas (1993) since 6000 BC. One can see that the most stationary parts of the record occurred precisely during the 370-year exceptional segments of solar motion denoted there by the dashed vertical lines and by three triangles.

\section{Predictive assessments for solar activity up to the year $2610 \mathrm{AD}$ based on analogies with the previous solar motion patterns}

Predictions of solar cycle heights and lengths have mostly been made on the basis of broad set of recent solar and geomagnetic data and on the basis of relations found only in the latest high activity solar cycles. Not knowing the underlying source of solar variability, such predictions can be false, as manifested at present in the case of the current cycle number 23 when a high to extremely high cycle has been predicted $\left(R_{\max }\right.$ within 140-225) (e.g. Wilson, 1992; Schatten et al., 1996; Kane, 1997). Ahluwalia (1999) predicted that cycle 23 will be a moderate cycle (more like cycle 17). However, it is now clear that the cycle 23 will be one of the lowest activity cycles of this century.

Solar motion, computable in advance, offers instead predictive possibilities, so far, of course, only as analogies with the results found for the previous six millennia. The solar orbits plotted in the intervals of 50 to 65 years from 1680 to 2001 (or 2135) are seen in Fig. 3b, 2. The Sun's orbit in the years 1985-2035 is of disordered (nontrefoil) type and similar to that of the second half of the nineteenth century. By analogy, mostly weaker and longer solar cycles should occur. Their lengths could vary between 9.6 and 12.3 years, their $R_{\max }$ could lie between 65 and 140 . The predictions of $R_{\max }$ for the cycle 23 made during the last decade vary between 140 and 225 (an unacceptable range). Our own prediction made on the basis of solar motion was the only opinion to express the opposite: i.e. a low solar cycle was predicted (Charvátová, 1988, 1990a, b; Charvátová and Střeštík, 1991). Charvátová (1990b) wrote: "The current cycle 22 is probably the last of the high ones. It should be followed by an epoch of about 40 years, in which the solar motion will be chaotic (disordered) and solar activity, therefore, should be low. The cycles will probably be longer and irregular." Charvátová (1995a, b) pointed out that the lengths of future cycles should be variable. As indicated by the initial, 3-year part of the cycle 23 in comparison with the respective part of the cycle 22 (in smoothed form, about $R_{\max } 80-90$ in comparison with $R_{\max } 155$ in the same time since the 

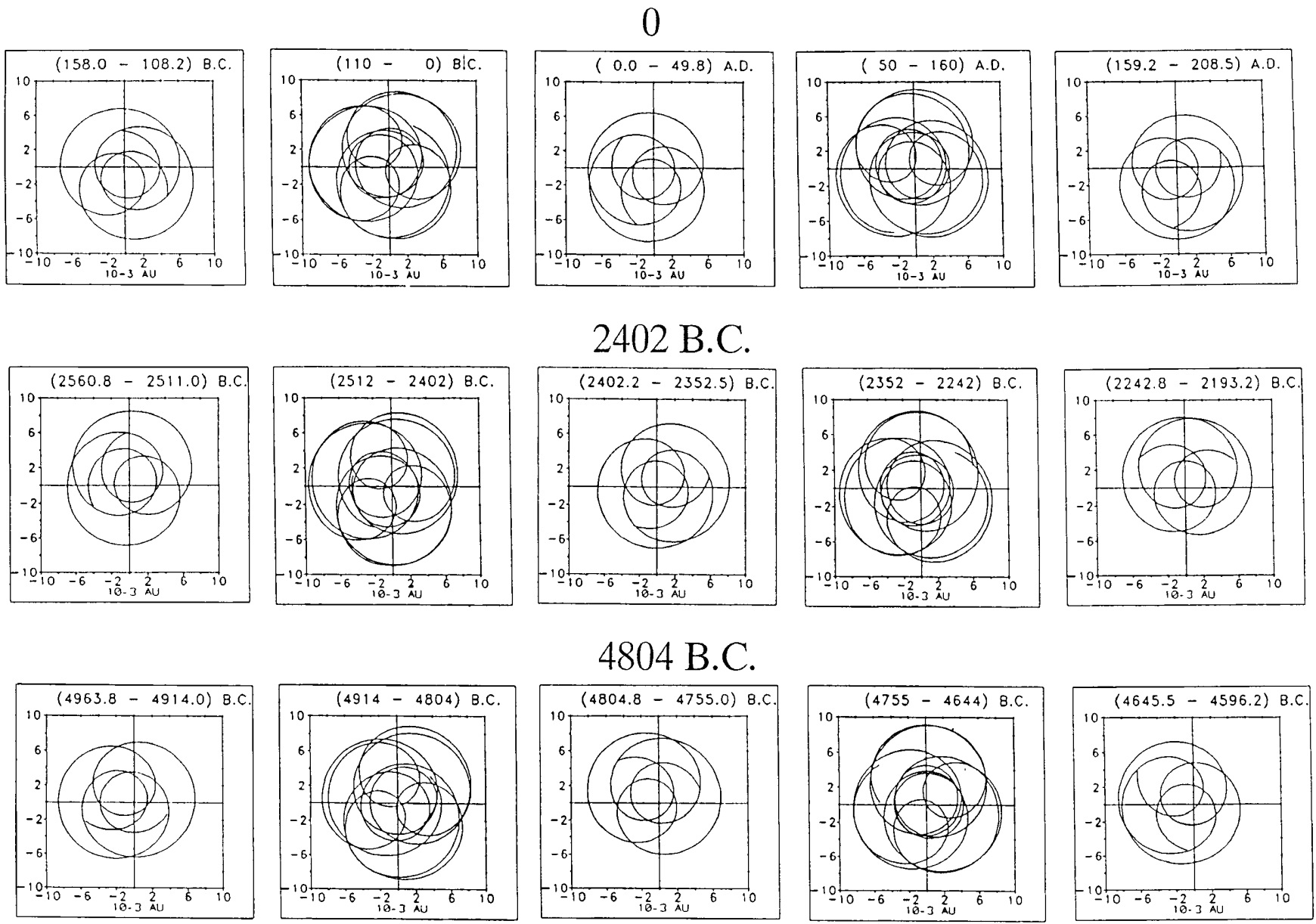

Fig. 4. The 370-year segments of the exceptional, stable pattern of solar motion recurring in steps of 2402 years: notice the twice shortened distance of 159 years between the three trefoils in each

minimum), our prediction was true. After $2085 \mathrm{AD}$, the Sun will move into the orbital trefoil. The activity series of the cycles 15-19 should be repeated.

The next 370-year exceptional segment will occur after AD 2240. Until 2610 AD, approximately stationary activity should occur as a repetition of solar behaviour during the previous exceptional segments (Figs. 5 and 6). High solar activity cycles with lengths of 10 years could prevail, corresponding to the 10-year lengths occurring for the trefoil type of solar motion.

\section{Conclusion}

These results show that the solar motion around the centre of mass of the solar system could be considered as the cause of solar variability. Not a static, but a dynamic Sun should be taken into account. It appears that solar motion is also the cause of the 2400-year cycle found in proxy of solar activity. The results indicate that the so far unconfirmed source of the approximately 2400-year cycle in solar activity, somewhat surprisingly, could be in solar motion.

The discovery of the exceptional 370-year segments of solar motion that recur in steps of 2402 years and segment (from $158 \mathrm{BC}$ to $208 \mathrm{AD}$, from $2561 \mathrm{BC}$ to $2193 \mathrm{BC}$ and from $4964 \mathrm{BC}$ to $4596 \mathrm{BC}$ ). The next such segment will occur between 2240 and $2610 \mathrm{AD}$

their imprint in the radiocarbon record is the main result presented here. The basic cycle of 178.7 years, being the interval between consecutive 50 -year orbital trefoils, is twice shortened by about 20 (JS) years, and during the intermediate intervals the Sun moves along the trefoil to a quasitrefoil orbit (Fig. 4). The 50-year orbital trefoils in steps of 178.7 years and the 370-year exceptional segments in steps of 2402 years represent the exceptional patterns of solar motion (Figs. 2, 4). A response of the 50 -year trefoil motion in solar activity is a series of five high, 10-year solar cycles (Fig. 3a). A response of the 370 -year segments of trefoil to quasitrefoil motion is approximately stationary (linear) with higher solar activity (Figs. 5 and 6). The 10-year cycle length prevails there.

Since solar motion is computable in advance, this permits predictive assessments for future solar behaviour. Moving along the disordered orbit to 2035 AD. (Fig. 2, bottom), which is similar to that of the second half of the nineteenth century (Fig. 3b), the Sun should develop lower solar cycles ( $R_{\max }$ from 65 to 140$)$ of very variable length (from 9.6 to 12.3 years). The initial development of the cycle 23, now in its third year, confirms this for the present cycle. Between 2086 and $2135 \mathrm{AD}$, a set of cycles comparable to 15 to 19 should be 

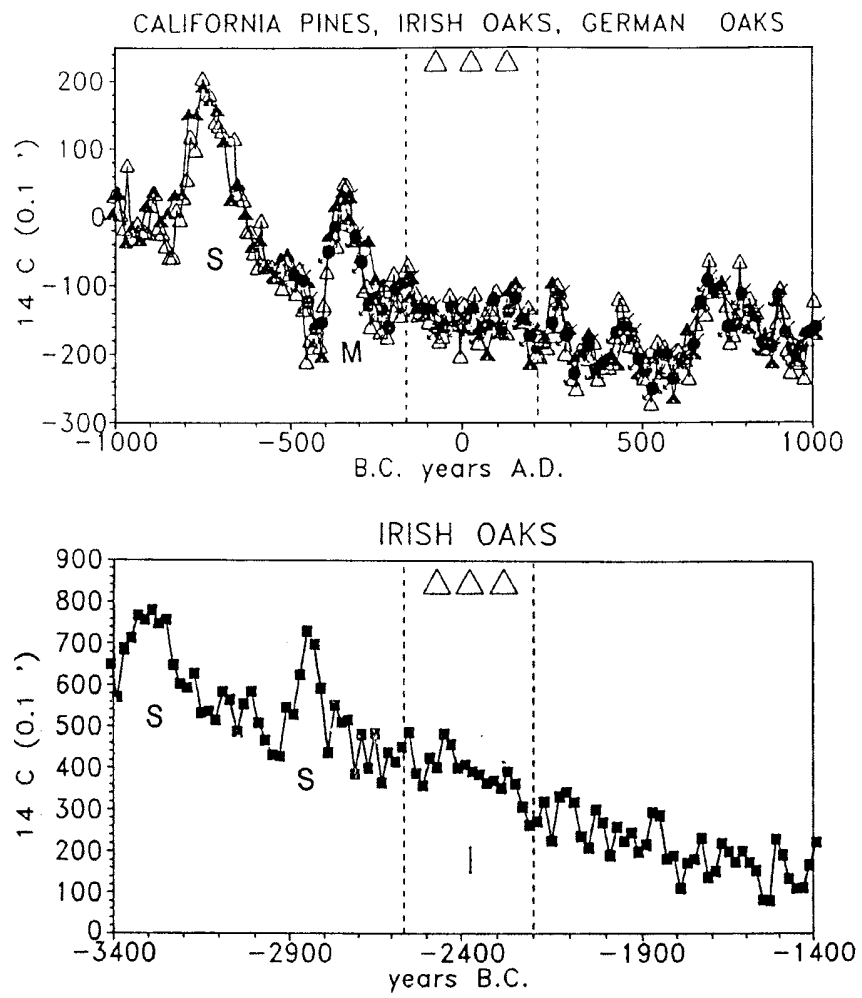

IRISH OAKS

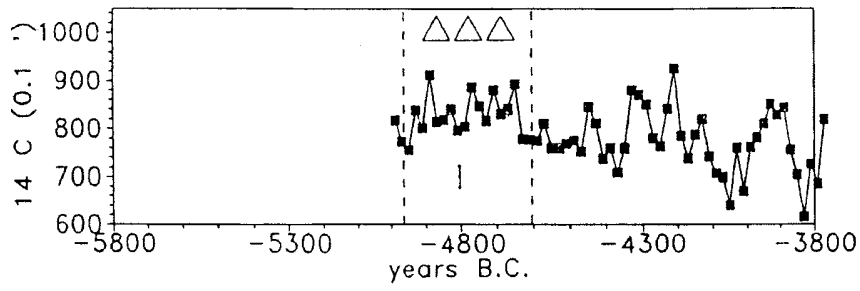

Fig. 5. Three intervals (1000 years preceding and following the centres of the 370-year exceptional segments). The radiocarbon data (decadal and bidecadal) of local or regional type was taken from Stuiver and Becker (1993), Stuiver and Pearson (1993) and Pearson and Quay (1993). The exceptional segments are denoted by the dashed vertical lines and three triangles. Error bars are plotted in the centres of the exceptional segments

repeated because the Sun will again move along a trefoil orbit.

Predictive assessments for the following centuries can be based on the 370-year exceptional segments and the related results. The next such segment will occur from AD 2240 to 2610. In correspondence with solar behaviour during the three previous exceptional 370-year segments, approximately stationary, high solar activity will be observed. The 10-year cycle lengths should prevail. A very long term maximum of solar activity comparable to that which was last observed during classical antiquity should occur in the mentioned interval (also see Stothers, 1979).

Statistical processing of the solar activity records in context with two types of solar motion formations is needed to estimate whether solar motion responds in solar activity and to understand mutual relations between the two phenomena. According to the results

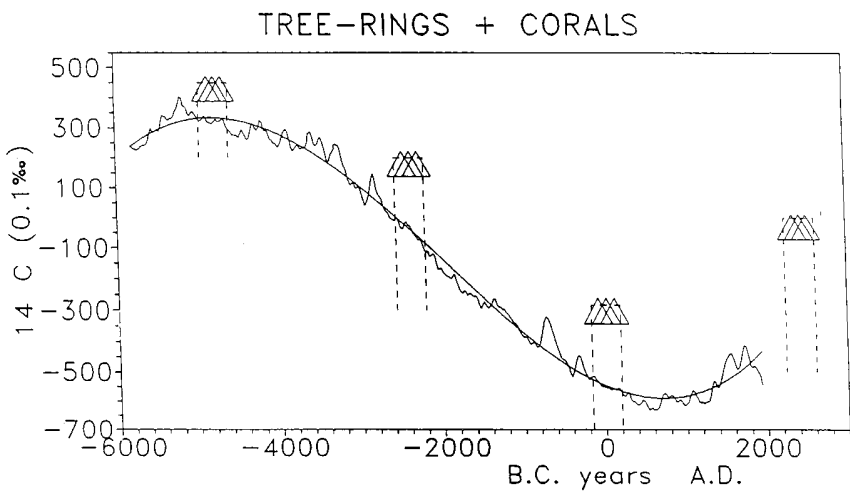

Fig. 6. The data are here plotted for the whole period since 6000 BC. (marine model ${ }^{14} \mathrm{C}$ ages calculated from the bidecadal atmospheric tree-ring data and a smoothing spline from coral data, Stuiver and Braziunas, 1993). The most stationary parts of the record coincide with the very exceptional segments of solar motion. They are denoted by the dashed vertical lines and by three triangles. The same type of record (approximately stationary, high solar activity) can be expected for the next exceptional segment which will occur between AD 2240 and 2610

obtained, the appropriate mechanism could eventually be established. A key to this is likely hidden in the 50 year trefoil interval. The mutual relations found between solar motion and solar activity and the latest knowledge about the internal structure of the Sun (obtained by SOHO, e.g. Turck-Chieze et al., 1997; Kosowichev et al., 1997) should be considered for this purpose. The individual solar spheres must surely respond to solar motion in distinct ways, provoking on the boundary layers a solar dynamo. The thin layer at the boundary between radiative and convection zones, where a shear flow was found by SOHO - MDI, 'is likely to be the place where the solar dynamo operates' (Kosowichev et al., 1997). The results indicate that 'solar dynamo' that was long sought in the solar interior, operates more likely from the outside, by means of the varying planetary configurations. As has been shown in Charvátová (1995a, b, c, 1997a), the solar motion could aid predictions also for terrestrial phenomena including climate.

Acknowledgements. This research was performed under the support of the Grant Agency of the Czech Republic, grant 97/205/ 0921.

Topical Editor E. Antonucci thanks R.W. Fairbridge and $\mathrm{G}$. Bonino for their help in evaluating this paper.

\section{References}

Ahluwalia, H. S., Present status of our prediction for solar cycle 23 maximum activity, in Proc. 26th Intern. Cosmic Ray Conf. (Salt Lake City, Utah, August 1999), 6, 260-263, 1999.

Charvátová, I., The solar motion and the variability of solar activity, Adv. Space Res., 8, (7) 147-150, 1988.

Charvátová, I., The relations between solar motion and solar variability, Bull. Astron. Inst. Czech., 41, 56-59, 1990a.

Charvátová, I., On the relation between solar motion and solar activity in the years $1730-80$ and 1910-60, Bull. Astron. Inst. Czech., 41, 200-204, 1990b. 
Charvátová, I., Repeating behaviour of solar and volcanic activities in the ordered intervals of solar inertial motion, in Proc. Workshop PAGES; Manifestations of climate at the Earth surface at the end of the Holocene, Eds. E. Růžičková, and A. Zeman Kolín, October 13-14th, 1994, 26-37, 1995a.

Charvátová, I., Solar-terrestrial and climatic variability during the last several millenia in relation to solar inertial motion, J. Coastal Res., Holocene Cycles, Climate, Sea Levels, and Sedimentation (Spec. Iss. 17), 343-354, 1995 b.

Charvátová, I., Solar-terrestrial variability in relation to solar inertial motion, Research Report of the Center for Theoretical Study at $C U$ and AS CR, CTS-95-04 and CTS-95-08 (2nd Edn), 24pp, 1995c.

Charvátová, I., Solar-terrestrial and climatic phenomena in relation to solar inertial motion, Surv Geophys., 18, 131-146, 1997a.

Charvátová, I., Solar motion (main article), in: Encyclopedia of planetary sciences, Eds. J. H. Shirley and R. W. Fairbridge, Chapman \& Hall, New York, 748-751, 1997b.

Charvátová, I., The basic cycles of 178.7 years and 2402 years in solar motion and their response in solar activity. JENAM 98, Solar Phys., Abstr, Prague, September 9-12th, p. 28, 1998.

Charvátová, I., and J. Střeštík, Solar variability as a manifestation of the Sun's motion, J. Atmos. Terr. Phys., 53, 1019-1025, 1991.

Charvátová, I., and J. Střeštík, The solar systemic features in STphenomena and surface air temperature during the last centuries, in: Contemporary climatology, Eds. R. Brázdil, and M. Koláŕ, Brno, 136-141, 1994.

Damon, P. E., and T. W. Linick, Geomagnetic-heliomagnetic modulation of atmospheric radiocarbon production, Radiocarbon, 28, 266-278, 1986.

Damon, P. E., and C. P. Sonett, Solar and terrestrial components of the atmospheric ${ }^{14} \mathrm{C}$ variation spectrum, in: The Sun in Time, Eds. C. P. Sonett, M. S. Giampapa and M. S. Matthews, The University of Arizona, Tucson, 360-388, 1991.

Damon, P. E., S. Cheng, and T. W. Linick, Fine and hyperfine structure in the spectrum of secular variations of atmospheric D ${ }^{14} \mathrm{C}$, Radiocarbon, 31, 704-718, 1989.

Fairbridge, R. W., Orbital commensurability and resonance, in: Encyclopedia of planetary sciences, Eds. J. H. Shirley and R. W. Fairbridge, Chapman \& Hall, London, 564-571, 1997.

Fairbridge, R. W., and J. E. Sanders, The Sun's orbit AD 750-2050. Basis for new perspectives on planetary dynamics and EarthMoon linkage, in: Climate, history and predictability, Eds. Rampino, M. R. et al., Van Nostrand-Reinhold New York, 446-471,1987.

Fairbridge, R. W., and J. H. Shirley, Prolonged minima and the 179-yr cycle of the solar inertial motion, Sol. Phys., 110, 191-220, 1987.
Hood, L. L., and J. L. Jirikowic, A probable 2400 year solar quasicycle in atmospheric D ${ }^{14} \mathrm{C}$. Holocene, 12, 98-105, 1991.

Jakubcová, I., and M. Pick, Correlation between solar motion, earthquakes and other geophysical phenomena, Ann. Geophysicae, 5B, 135-141, 1987.

Jose, P. D., Sun's motion and sunspots, Astron. J., 70, 193-200, 1965.

Kane, R. P., A preliminary estimate of the size of the coming solar cycle 23, based on Ohl's precursor method, Geophys. Res. Lett., 24, 1899-1902, 1997.

Kosowichev, A. G., J. Schou, P. H. Scherer, R. S. Bogart, R. I. Bush, J. T. Hoeksema, J. Aloise, L. Bacon, A. C. Burnette, P. M. De Forest, K. Giles, R. Leibrand, M. Nigam, M. Rubin, K. Scott, and S. D. Williams, Structure and rotation of the solar interior: Initial results from the MDI Medium-L program, in: The first results from $\mathrm{SOHO}$, Eds. B. Fleck and Z. Svestka, Kluwer Dordrecht, 43-61, 1997.

Pearson, G. W., and F. Quay, High precision ${ }^{14} \mathrm{C}$ measurement of irish oaks to show ${ }^{14} \mathrm{C}$ variations from $\mathrm{AD} 1840-5000$ BC: a correction, Radiocarbon, 35, 105-123, 1993.

Rabin, D., M. R. Wilson, and R. L. Moore, Bimodality of the solar cycle, Geophys. Res. Lett., 13, 352-354, 1986.

Shatten, K. H., D. J. Myers and S. Sofia, Solar aktivity forecast for solar cycle 23, Geophys. Res. Lett., 23, 605-608, 1996.

Sonett, C. P., and S. A. Finney, The spectrum of radiocarbon, Philos Trans. R. Soc. London. A 330, 413-426, 1990.

Stothers, R., Solar activity cycles during classical antiquity. Astron. Astrophys., 77, 121-127, 1979.

Stuiver, M., and B. Becker, High precision decadal calibration of the radiocarbon time scale AD 1950-6000 BC, Radiocarbon, 35, 35-65, 1993.

Stuiver, M., and T. F. Braziunas, Modeling atmospheric ${ }^{14} \mathrm{C}$ influences and ${ }^{14} \mathrm{C}$ ages of marine samples to $10000 \mathrm{BC}$, Radiocarbon, 35, 137-189, 1993.

Stuiver, M., and G. W. Pearson, High-precision bidecadal callibration of the radiocarbon time scale $\mathrm{AD} 1950-500 \mathrm{BC}$ and 2500-6000 вС, Radiocarbon, 35, 1-24, 1993.

Suess, H. E., The radiocarbon record in tree rings of the last 8000 years, Radiocarbon, 22, 200-209, 1980.

Turck-Chieze, S., S. Basu, A. S. Brun, J. Christensen-Dalsgaard, A. Eff-Darwich, I. Lopez, F. Pérez Hernandez, G. Berthomieu, J. Provost, L. K. Ulrich, F. Baudin, P. Boumier, J. Charra, A. H. Gabriel, R. A. Garcia, G. Grec, C. Renaud, J. M. Robillot, and T. Roca Cortes, First view of the solar core from GOLF acoustic modes. in: The first results from SOHO, Eds., B. Fleck and Z. Švestka, Kluwer, Dordrecht, 247-265, 1997.

Wilson, R. M., Bimodality and the Hale cycle, Sol. Phys., 117, 269278, 1988.

Wilson, R. M., An early estimate for the size of cycle 23, Sol. Phys., 140, 181-193, 1992. 